\title{
Coevolutionary Free Lunches
}

\author{
David H. Wolpert, dhw@email.arc.nasa.gov \\ William G. Macready, wgm@email.arc.nasa.gov \\ NASA Ames Research Center \\ Moffett Field, CA, 94035
}

\begin{abstract}
Recent work on the foundations of optimization has begun to uncover its underlying rich structure. In particular, the "No Free Lunch" (NFL) theorems [WM97] state that any two algorithms are equivalent when their performance is averaged across all possible problems. This highlights the need for exploiting problem-specifc knowledge to achieve better than random performance. In this paper we present a general framework covering most search scenarios. In addition to the optimization scenarios addressed in the NFL results, this framework covers multi-armed bandit problems and evolution of multiple co-evolving agents. As a particular instance of the latter, it covers "self-play" problems. In these problems the agents work together to produce a champion, who then engages one or more antagonists in a subsequent multi-player game. In contrast to the traditional optimization case where the NFL results hold, we show that in self-play there are free lunches: in coevolution some algorithms have better performance than other algorithms, averaged across all possible problems. However in the typical coevolutionary scenarios encountered in biology, where there is no champion, NFL still holds.
\end{abstract}

\section{INTRODUCTION}

Optimization algorithms have proven to be valuable in almost every setting where quantitative £gures of merit are available. Recently, the mathematical foundations of optimization have begun to be uncovered [KWM01], [MW96], [FHS01], [IT01], [CO01]. One particular result in this work, the "No Free Lunch" (NFL) theorems, establishes the equivalent performance of all optimization algorithms when averaged across all possible problems [KWM01]. Numerous works have extended these early results, and considered their application to different types of optimization (e.g. to multi-objective optimization [CK03]). The web site www. no-free-lunch. org offers a list of recent references.

However, all previous work has been cast in a limited manner that does not cover repeated game scenarios where the fgure of merit can vary based on the response of another player. In particular, the NFL theorems do not cover such scenarios. These game-like scenarios are usually called "coevolutionary" since they involve the behaviors of more than a single agent or player [FP00].

One important example of coevolution is "self-play", where the players cooperate to train one of them as a champion. That champion is then pitted against an antagonist in a subsequent multi-player game. The goal is to train that champion to perform as well as possible in that subsequent game. For a checkers example see [CF99]. We will refer to all players other than the one of direct attention as that player's "opponents", even when (as in self-play) the players are actually cooperating. (Sometimes when discussing self-play we will refer to the specifc opponent to be faced by a champion in a subsequent game - an opponent not under our control - as the champion's "antagonist".)

Coevolution can also be used for problems that on the surface appear to have no connection to a game (for an early application to sorting networks see [Hil92]). Coevolution in these cases enables escape from poor local optima in favor of better local optima.

In this paper we frst present a mathematical framework that covers both traditional optimization and coevolutionary scenarios. (It also covers other scenarios like multi-armed bandits.) We then use that framework to explore the differences between traditional optimization and coevolution. We fnd dramatic differences between the traditional optimization and coevolutionary scenarios. In particular, unlike the fundamental NFL result for traditional optimization, in the self-play domain there are algorithms which are superior to other algorithms for all problems. However in the typical coevolutionary scenarios encountered in biology, where there is no champion, NFL still holds.

\section{GENERAL FRAMEWORK}

In this section we present a general framework, and illustrate it on two examples. Despite its substantially greater breadth of applicability, the formal structure of this framwork is only a slight extension of that used in [WM97].

\section{A. Formal framework specifcation}

Say we have two spaces, $X$ and $Z$. To guide the intuition, a typical scenario might have $x \in X$ be the joint strategy followed by our player(s), and $z \in Z$ be the probability distribution over some space of possible rewards/payoffs to the champion, or over possible £gures of merit, or some such.

In addition to $X$ and $Z$, we also have a ftness function

$$
f: X \mapsto Z \text {. }
$$

In the example where $z$ is a probability distribution over rewards, $f$ can be viewed as the specifcation of an $x$ conditioned probability distribution of rewards.

We have a total of $m$ time-steps, and represent the information generated through those time-steps as

$$
d_{m} \equiv\left(d_{m}^{x}, d_{m}^{z}\right) \equiv\left(\left\{d^{x}(t)\right\}_{t=1}^{m},\left\{d^{z}(t)\right\}_{t=1}^{m}\right)
$$

Each $d^{x}(t)$ is a particular $x \in X$. Each $d^{z}(t)$ is a (perhaps stochastic) function of $f\left(d_{X}\left(t^{\prime}\right)\right)$. For example, say $z$ 's values of $f(x)$ - are probability distributions over reward values. Then $d^{z}(t)$ could consist of the full distribution $f\left(d^{x}(t)\right)$. 
Alternatively, it could consist of a moment of that distribution, or even a random sample of it. In general, we allow the function specifying $d^{z}(t)$ to vary with $t$, although that freedom will not be exploited here. As shorthand we will write $d(t)$ to mean the pair $\left(d^{x}(t), d^{z}(t)\right)$.

A search algorithm $a$ is an initial distribution $P_{1}\left(d^{x}(1)\right)$, together with a set of $m-1$ separate conditional distributions $P_{t}\left(d^{x}(t) \mid d_{t-1}\right), t=2, \ldots m$. Such an algorithm specifes what $x$ to choose, based on the information uncovered so far, for any time-step $t$. Finally, we have a vector-valued cost function, $C\left(d_{m}, f\right)$ which we use to assess the performance of the algorithm. Often our goal is to fnd the $a$ that will maximize $E(C)$, for a particular choice of how to form the $d^{z}(t)$ 's.

The NFL theorems concern averages over all $f$ of quantities involving $C$. For those theorems to hold, for $f$-averages of $C$ to be independent of the search algorithm, it is crucial that $C$ does not depend on $f$. (The framework in [WM97] defnes cost functions as real-valued functions of $d_{m}$.) When that independence is relaxed, the NFL theorems need not hold. Such relaxation occurs in self-play, for example, and is how one can have free lunches in self-play. This papers explores this phenomenon.

\section{B. Examples of the framework}

a) Example 1: One example of this framework is the scenario considered in the NFL theorems. There each $z$ is a probability distribution over a space $Y \subseteq \mathbb{R}$. For convenience we take $X$ and $Y$ countable. Each $d_{f}(t)$ is a sample of the associated function $z(t)=f\left(d^{x}(t)\right)$. The search algorithm is constrained so that

$$
P_{t}\left(d^{x}(t)=x \mid d_{t-1}\right)=0 \quad \forall x \in d_{t-1}^{x},
$$

i.e., so that the search never revisits points already sampled. ${ }^{1}$ Finally, $C\left(d_{m}, f\right)$ is allowed to be any scalar-valued function that depends on $d_{m}$ exclusively.

The NFL theorems apply to any scenario meeting these specifcations.

b) Example 2: Another example is the multi-arm bandit problem introduced for optimization by Holland [Hol75] and thoroughly analyzed in [MW98]. The scenario for that problem is identical to that for the NFL results, except that there are no constraints on the search algorithm, $Y=\mathbb{R}$ and every $z$ is a Gaussian. The fact that revisits are allowed means that NFL need not apply.

c) Example 3: Self-play is identical to the NFL scenario except that $C$ depends on $f$. This dependence is based on a function $A\left(d_{m}\right)$ mapping $d_{m}$ to a subset of $X$. Intuitively, $A$ specifes the details of the champion, based on the $m$ repeated games and on the possible responses to the champion of an antagonist in a subsequent game. $C$ is then based on this specifcation of the champion. Formally, it uses $A$ to

\footnotetext{
'This requirement is just to "normalize" algorithms. In general, an algorithm that sometimes revisits points can outperform one that never does. Our requirement simply says that we're purely focusing on how well the algorithms choose new points, not how smart they are about whether to Enish the search at $t=m$ by sampling a new point or by returning to one already visited. See [WM97].
}

determine the quality of the search algorithm that generated $d_{m}$ as follows:

$$
C\left(d_{m}, f\right)=\min _{x \in A\left(d_{m}\right)} \mathbb{E}_{f(x)}
$$

where $\mathbb{E}_{f(x)}$ is the expected value of the distribution of payoffs $f(x)$ obtained from $x, \sum_{y \in Y} y P_{f}(y \mid x)=\sum_{y \in Y} y[f(x)](y)$. Intuitively, this measure is the worst possible payoff to the champion.

To see in more detail how this describes self-play, assume two players, with strategy spaces $X_{1}$ and $X_{2}, X_{1}$ being the strategy space of our champion. Take $x$ to be the joint strategy of our players in any particular game, i.e., $x \in X=X_{1} \times X_{2}$. So $d_{m}^{x}$ specifes the $m$ strategies followed by our champion (as well as that of the other player) during the $m$ training games. $d_{m}^{z}$ is the associated set of rewards to our champion, i.e., each $d^{z}(t)$ is a sample of the distribution $f\left(d^{x}(t)\right)$.

Let $x_{1} \in X_{1}$ be the strategy our champion elects to follow based on that training data. Notc that that strategy can be represented as the set of all joint-strategies $x$ whose frst component is $x_{1}$. We adopt this representation, and write the strategy chosen by our champion - the set of all $x$ 's consistent with the champion's choice of strategy $x_{1}-$ as $A\left(d_{m}\right) \subset X$.

Say the antagonist our champion will now face is able to choose the worst possible element of $X_{2}$ (as far as expected reward to our champion is concerned), given that our champion chooses strategy $A\left(d_{m}\right)$. If the antagonist does this the expected reward to our champion is given by $C\left(d_{m}, f\right)$ as defned above. Obvious variants of this setup replace the worst-case nature of $C$ with some alternative, have $A$ be stochastic, etc. Whatever variant we choose, typically our goal in self-play is to choose $a$ and/or $A$ so as to maximize $E(C)$, the expectation being over all possible $d_{m}$. The fact that $C$ depends on $f$ means that NFL need not apply. The mathematical structure that replaces NFL is explored in the following sections of this paper.

d) Example 4: The basic description of self-play in the introduction looks like a special case of the more general biological coevolution scenario. However in terms of our framework they are quite different.

In the general coevolution scenario there are a total of $N$ agents (or players, or species', or genes, etc). Their strategy spaces are written $X_{i}$, as in self-play. Now though $X$ is extended beyond the current joint strategy, to include the previous joint "population frequency" value. Formally, we write

$$
X=\left(X_{1}, u_{1}\right) \times \cdots \times\left(X_{N}, u_{N}\right),
$$

and interpret each $u_{i} \in \mathbb{R}$ as agent $i$ 's previous population frequency. As explained below, the reason for this extension of $X$ is so that $a$ can give the sequence of joint population frequencies that accompanies the sequence of joint strategies.

In the general coevolution scenario each $z$ is a probability distribution over the possible current population frequencies of the agents. So given our defnition of $X$, we interpret $f$ as a map taking the previous joint population frequency, together with the current joint strategy of the agents, into a probability distribution over the possible current joint population frequencies of the agents. 
As an example, in evolutionary game theory, the joint strategy of the agents at any given $t$ determines the change in tach one's population frequency in that time-step. Accordingly, in the replicator dynamics of evolutionary game theory, $f$ takes a joint strategy $x_{1} \times \ldots x_{N}$ and the values of all agents' previous population frequencies, and based on that determines the new value of each agent's population frequency.

As before, each $d^{z}(t)$ contains the information coming out of $f\left(d^{x}(t)\right)$. Here that information is the set of current population frequencies. The search algorithm a now plays two roles. One of these is to directly incorporate those current population frequencies into the $\left\{u_{i}\right\}$ components of $d^{x}(t+1)$. The other is, as before, to determine the joint strategy $\left[x_{1}, \ldots, x_{N}\right]$ for time $t+1:$ As in self-play, this strategy of each agent $i$ is given by a (potentially stochastic and/or time-varying) function $a_{i}$. An application of $a$ is given by the simultaneous operation of all those $N$ distinct $a_{i}$ on a common $d_{t}$, as well as the transfer of the joint population frequency from $d^{z}(t)$, to produce $d^{x}(t+1)$.

Note that the choice of joint strategy given by a may depend on the previous time-step's frequencies. As an example, this corresponds to sexual reproduction in which mating choices are random. ${ }^{2}$ However in the simplest version of evolutionary game theory, the joint strategy is actually constant in time, with all the dynamics occuring via frequency updating in $f$. If the agents are identifed with distinct genomes, then in this version reproduction is parthenogentic.

Finally, $C$ is now a vector with $N$ components, each component $j$ only depending on the associated $d_{t}(j)$. In general in biological coevolution scenarios (e.g., evolutionary game theory), there is no notion of a champion being produced by the search and subsequently pitted against an antagonist in a "bake-off". Accordingly, there is no particular signifcance to results for $C$ 's that depend on $f$.

This means that so long as we make the approximation, reasonable in real biological systems, that $x$ 's are never revisited, all of the requirements of Ex. 1 are met. This means that NFL applies. So in particular, say we restrict attention to the particular kinds of $a$ 's of evolutionary game theory. Then any two choices of $a$ - any two sets of strategy-making rules $\left\{a_{i}\right\}$ - perform just as well as one another, averaged over all $f$ 's. More generally, we can consider other kinds of $a$ as well, and the result still holds.

\section{APPLICATION TO SELF-PLAY}

In example 3 of section II-B we introduced self-play model. In the remainder of this paper we show how free lunches may arise in this setting, and quantify the a priori differences between certain self-play algorithms. For expository simplicity, we modify the defnitions introduced in the general framework, to tailor them for self-play.

In self-play agents (or game strategies) are paired against each other in a (perhaps stochastically formed) sequence to generate a set of 2-player games. After $m$ distinct training

${ }^{2}$ Obvious elaborations of the framework allow $x$ to include relative rewards from the preceding round, as well as frequencies. This allows mate selection to be based on current differential ftness, as well as overall frequency in the population. games between an agent and its opponents, the agent enters a competition Performance of the agent is measured with a payoff function. As shorthand, the (here deterministic) payotf function when the $i$ th agent plays move (strategy) $\underline{x}_{i}$ and $i$ 's opponent plays $\bar{x}_{i}$ is written as $f_{i}\left(\underline{x}_{i}, \bar{x}_{i}\right)$. If we indicate the joint move of $i$ and its opponent as $x_{i}=\left(\underline{x}_{i}, \bar{x}_{i}\right)$ we can write the payoff to agent $i$ as $f_{i}\left(x_{i}\right)$. In the following we make no assumption about the structure of moves except that they are Enite. $\underline{x}$ might represent a sequence of plays representing an entire game of checkers and $\bar{x}$ might represent a complete set of opponent responses to each play. The payoff function $f(\underline{x}, \bar{x})$ might then represent the outcome of the game as +1 for a win for $i, 0$ for a draw, and -1 for a loss. nlegal joint moves can be eliminated by appropriately limiting the space of moves and opponent responses in order to satisfy the rules of the game. In other applications, $\underline{x}$ might represent an algorithm to sort a list and $\bar{x}$ a mutable set of lists to be sorted. The payoff would then reaect the ability of the algorithm to sort those lists in $\bar{x}$.

We defne the payoff for agent $i$ playing move $x_{i}$ independent of an opponent's reply, $g\left(\underline{x}_{i}\right)$, as the least payoff over all possible opponent responses (a minimax criteria): $g_{i}\left(\underline{x}_{i}\right) \equiv \min _{\bar{x}_{i}} f_{i}\left(\underline{x}_{i}, \bar{x}_{i}\right)$. With this criterion, the best move an agent can make is that move which maximizes $g_{i}$ so that its performance in competition (over all possible opponents) will be as good as possible. We are not interested in search strategies just across $i$ 's possible moves, but more generally across all joint moves of $i$ and its opponents. (Note that whether that opponent varies or not is irrelevant, since we are setting its moves.) The ultimate goal is to maximize i's minimax performance $g_{i}$.

We make one important observation. In general, using a random pairing strategy in the training phase will not result in a training set that can be used to guarantee that any particular move in the competition is better than the worst possible move. The only way to ensure an outcome guaranteed to be better than the worst possible is to exhaustively explore all possible responses to move $\underline{x}$, and then determine that the worst value of $f_{i}$ for all such joint moves is better than the worst value for some other move, $\underline{x}_{i}^{\prime}$. To do this certainly requires that $m$ is greater than the total number of possible moves by the opponent but even for very large $m$ unless all possible opponent responses have been explored we can not make any such guarantees.

Pursuing this observation further, consider the situation where we know (perhaps through exhaustive sampling of opponent responses) that the worst possible payoff for some move $\underline{x}$ is $g(\underline{x})$ and that another joint move $x^{\prime}=\left(\underline{x}^{\prime}, \bar{x}^{\prime}\right)$ with $\underline{x} \neq \underline{x}^{\prime}$ results in a payoff $f\left(x^{\prime}\right)<g(\underline{x})$. In this case there is no need to explore other opponent responses to $\underline{x}^{\prime}$ since it must be that $g\left(\underline{x}^{\prime}\right)<g(\underline{x})$, i.e. $\underline{x}^{\prime}$ is minimax inferior to $\underline{x}$. Thus, considering strategies for searching across the space of joint moves $x_{i}$, any algorithm that avoids searching regions which are known to be minimax inferior (as above) will be more effcient than one which searches these regions (e.g. random search). This applies for all $g_{i}$ and so the smarter algorithm will have an average performance greater than the dumb algorithm. Very roughly speaking, this result avoids NFL 
implications because uniformly varying over all $g_{i}$ does not uniformly vary over all possible $f_{i}$, which are the functions that ultimately determines performance.

In the following sections we explore this observation further.

\section{A. Defnitions}

As much as possible we follow the notation of [WM97] extending it where necessary. That paper should be consulted as motivation for the analysis framework we employ. Without loss of generality we now consider two player games, and leave the agent index $i$ implicit. If there are $\underline{l}$ moves available to an agent, we label these by $\underline{x} \in \underline{X} \equiv[1, \cdots, \underline{l}]$. For each such move we assume the opponent may choose from one of $\bar{l}(\underline{x})$ possible moves forming the space $\bar{X}(\underline{x}) .{ }^{3}$ For simplicity we will take $\bar{X}(\underline{x})$ to be independent of $\underline{x}$. Consequently, the size of the joint move space is $|X|=\sum_{x=1}^{\underline{l}} \bar{l}(\underline{x})$. If the training period consists of $m$ distinct joint moves, even with $m$ as large as $|X|-\underline{l}$, we cannot guarantee that the agent won't choose the worst possible move in the competition as the worst possible move could be the opponent response that was left unexplored for each of the $\underline{l}$ possible moves.

In [WM97] a population is a sample of distinct points from the input space $X$ and their corresponding ftness values. In this coevolutionary context the notion of a population of sampled confgurations needs to be extended to include opponent responses. For simplicity we assume that ftness payoffs are a deterministic function of joint moves. Thus, rather than the more general output space $Z$, we assume payoff values lie in a fnite totally ordered space. Consequently, the ftness function is the mapping $f: X \mapsto Y$ where $X=\underline{X} \times \bar{X}$ is the space of joint moves. As in the general framework a population of size $m$ is represented as

$$
d_{m}=\left\{\left(d_{m}^{x}(1) ; d_{m}^{y}(1)\right), \cdots,\left(d_{m}^{x}(m) ; d_{m}^{y}(m)\right)\right\}
$$

where $d_{m}^{x}(i)=\left\{d_{m}^{\underline{x}}(i), d_{m}^{\bar{x}}(i)\right\}$ and $d_{m}^{y}(i)=f\left(d_{m}^{\underline{x}}(i), d_{m}^{\bar{x}}(i)\right)$ and $i \in[1, \cdots, m]$ labels the samples taken. In the above defnition $d_{m}^{\frac{x}{m}}(i)$ is the $i$ th move made by the agent, $d_{m}^{\bar{x}}(i)$ is the opponent response, and $d_{m}^{y}(i)$ is the corresponding payoff. As usual we assume that no joint confgurations are revisited. A particular coevolutionary optimization task is specifed by defning the payoff function that is to be extremized. As discussed in [WM97] a class of problems is defned by specifying a probability density $P(f)$ over the space of possible payoff functions. As long as both $X$ and $Y$ are Enite (as they are in any computer implementation) this is straightforward.

In addition to this extended notion of a population, there is an additional consideration in the coevolutionary setting, namely the decision of what move to make in the competition based upon the results of the training population. Formally, we encapsulate the process of making this decision as $\underline{A} .^{4} \underline{A}$ consists of a set of distributions (one for each $m$ since we would like $\underline{A}$ to select a move regardless of the size of the

\footnotetext{
${ }^{3}$ Note that the space of opponent moves varies with $\underline{x}$. This is the typical situation in applications to games with complex rules (e.g. checkers).

${ }^{4}$ The notation $\underline{A}$ is meant to suggest that, unlike the $A\left(d_{m}\right)$ function introduced earlier, $\underline{A}$ defnes only the champions move, and not the possible responses to this move.
}

training set) of the form $P\left(\underline{x} \in \underline{X} \mid d_{m}\right)$. If $A$ deterministically returns a single move, we indicate the mapping from training population to move as $\underline{A}\left(d_{m}\right)$. To summarize, the defnition of search method is extended for self-play to include:

- A search rule $a$ which determines the manner in which a population is expanded during training and is formally given by the set of distributions $\left\{P_{t}\left(d^{x}(t) \mid d_{t-1}\right)\right\}_{t=1}^{m}$. This corresponds exactly to the defnition of a search algorithm in [WM97] used in non-coevolutionary optimization.

- A move-choosing rule $\underline{A}$ mapping probabilistically or deterministically to the single move used in the competition. We write $\underline{A}$ explicitly as the probability density $\underline{A}\left(\underline{x} \mid d_{m}\right)$ where $\underline{x} \in \underline{X}$. For deterministic $\underline{A}$ we write the density as $\underline{A}\left(\underline{x} \mid d_{m}\right)=\delta\left(\underline{x}-\underline{A}\left(d_{m}\right)\right)$.

The tuple $(a, \underline{A})$ is called a search process (as opposed to a search algorithm in [WM97]).

The search process seeks a strategy that will perform well in competition. If $\underline{A}$ is deterministic the natural measure of the performance of search process $(a, \underline{A})$ obtained during training is $C=\min _{i \in[1, m]} f\left(\underline{A}\left(d_{m}\right), d_{m}^{\bar{x}}(i)\right)$. (If $\underline{A}$ is not deterministic then we use the weighted average $\sum_{x \in X} \min _{i \in[1, m]} f\left(\underline{x}, d_{m}^{\bar{x}}(i)\right) \underline{A}\left(\underline{x} \mid d_{m}\right)$.) The best $(a, \underline{A})$ for a particular $f$ are those which maximize $C$.

The traditional version of NFL (for traditional optimization) defnes the performance differently since there is no opponent. In the simplest case the performance of $a$ (recall that there is no choosing algorithm) might be measured as $C=\max _{i \in[1, m]} d_{m}^{y}$. One traditional NFL result states that the average performance of any pair of algorithms is identical, or formally, $\sum_{f} P(C \mid f, m, a)$ is independent of $a .^{5} \mathrm{~A}$ natural extension of this previous results considers a nonuniform average over ftness functions. In this case the quantity of interest is $\sum_{f} P(C \mid f, m, a) P(f)$ where $P(f)$ weights different $£$ tness functions. NFL results can be proven for other non-uniform $P(f)$ [SVW01].

A result akin to this one in the self-play setting would state that the unform average $\sum_{f} P(C \mid f, m, a, \underline{A})$ is independent of $a$ and $\underline{A}$. However, as we have informally seen, such a result cannot hold in general since a search process with an $a$ that exhausts an opponent's repertoire of moves has better guarantees than other search processes. A formal proof of this statement is presented in the next section.

\section{AN INTUITIVE EXAMPLE}

Before proving the existence of free lunches we give a motivating example to both illustrate the defnitions made in the above section and to show why we might expect free lunches to exist. Consider the concrete case where the player has two possible moves, i.e. $\underline{X}=\{1,2\}$, the opponent has two responses for each of these moves, i.e. $\bar{X}=\{1,2\}$, and there are only two possible payoff values, i.e. $Y=\{1 / 2,1\}$. In this simple case there are 16 possible functions and these are listed in Table I. We can see that in this simple example the minimax

\footnotetext{
${ }^{5}$ Actually far more can be said, and the reader is encouraged to consult [WM97] for details.
} 


\begin{tabular}{|c|c|c|c|c|c|c|c|c|c|c|c|c|c|c|c|c|}
\hline$(\underline{x}, \bar{x})$ & $f_{1}$ & $f_{2}$ & $f_{3}$ & $f_{4}$ & $f_{5}$ & $f_{6}$ & $f_{7}$ & $f_{8}$ & $f_{9}$ & $f_{10}$ & $f_{11}$ & $f_{12}$ & $f_{13}$ & $f_{14}$ & $f_{15}$ & $f_{16}$ \\
\hline$(\underline{1}, 1)$ & $1 / 2$ & 1 & $1 / 2$ & 1 & $1 / 2$ & 1 & $1 / 2$ & 1 & $1 / 2$ & 1 & $1 / 2$ & 1 & $1 / 2$ & 1 & $1 / 2$ & 1 \\
$(1,2)$ & $1 / 2$ & $1 / 2$ & 1 & 1 & $1 / 2$ & $1 / 2$ & 1 & 1 & $1 / 2$ & $1 / 2$ & 1 & 1 & $1 / 2$ & $1 / 2$ & 1 & 1 \\
$(2,1)$ & $1 / 2$ & $1 / 2$ & $1 / 2$ & $1 / 2$ & 1 & 1 & 1 & 1 & $1 / 2$ & $1 / 2$ & $1 / 2$ & $1 / 2$ & 1 & 1 & 1 & 1 \\
$(2,2)$ & $1 / 2$ & $1 / 2$ & $1 / 2$ & $1 / 2$ & $1 / 2$ & $1 / 2$ & $1 / 2$ & $1 / 2$ & 1 & 1 & 1 & 1 & 1 & 1 & 1 & 1 \\
\hline$\underline{x}$ & $g_{1}$ & $g_{2}$ & $g_{3}$ & $g_{4}$ & $g_{5}$ & $g_{6}$ & $g_{7}$ & 98 & $g_{9}$ & $g_{10}$ & $g_{11}$ & $g_{12}$ & $g_{13}$ & $g_{14}$ & $g_{15}$ & $g_{16}$ \\
\hline 1 & $1 / 2$ & $1 / 2$ & $1 / 2$ & 1 & $1 / 2$ & $1 / 2$ & $1 / 2$ & 1 & $1 / 2$ & $1 / 2$ & $1 / 2$ & 1 & $1 / 2$ & $1 / 2$ & $1 / 2$ & 1 \\
2 & $1 / 2$ & $1 / 2$ & $1 / 2$ & $1 / 2$ & $1 / 2$ & $1 / 2$ & $1 / 2$ & $1 / 2$ & $1 / 2$ & $1 / 2$ & $1 / 2$ & $1 / 2$ & 1 & 1 & 1 & 1 \\
\hline
\end{tabular}

TABLE I

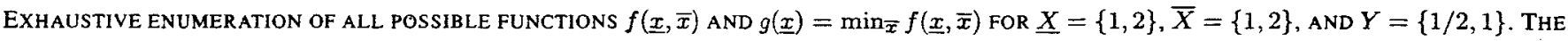
PAYOFF FUNCTIONS LABELED IN BOLD ARE THOSE CONSISTENT WITH THE POPULATION $d_{2}=\{(1,2 ; 1 / 2),(2,2 ; 1)\}$.

criteria gives a very biased distribution over possible performance measures: $9 / 16$ of the functions have $g=\left[\begin{array}{ll}1 / 2 & 1 / 2\end{array}\right]$, $3 / 16$ have $g=\left[\begin{array}{ll}1 / 2 & 1\end{array}\right], 3 / 16$ have $g=\left[\begin{array}{ll}1 & 1 / 2\end{array}\right]$, and $1 / 16$ have $g=\left[\begin{array}{ll}1 & 1\end{array}\right]$ where $g=[g(\underline{x}=1) \quad g(\underline{x}=2)]$.

If we consider a particular population, say $d_{2}=$ $\{(1,2 ; 1 / 2),(2,2 ; 1)\}$, the payoff functions that are consistent with this population are $f_{9}, f_{10}, f_{13}, f_{14}$ and the corresponding distribution over $g$ functions is $1 / 2\left[\begin{array}{ll}1 / 2 & 1 / 2\end{array}\right]^{\top}$ and $1 / 2\left[\begin{array}{ll}1 / 2 & 1\end{array}\right]^{\top}$. Given the fact that any population will give a biased sample over $g$ functions it may not surprising that there are free lunches. We might expect that an algorithm which is able to exploit this biased sample would perform uniformly better than another algorithm which does not exploit the biased sample of $g s$. In the next section we prove the existence of free lunches by constructing such a pair of algorithms.

\section{ProOF OF FreE LUNCHES}

In this section a proof is presented that there are free lunches for self-play by constructing a pair of search processes one of which explicitly has performance equal to or better than the other for all possible payoff functions $f$. As in earlier NFL work we assume that both $|X|$ and $|Y|$ are fnite. ${ }^{6}$ For convenience, and with no loss in generality, we normalize the possible $Y$ values so that they are equal to $1 /|Y|, 2 /|Y|, \cdots, 1$.

The pair of processes we construct use the same search rule $a$ (it is not important in the present context what $a$ is) but different deterministic move choosing rules $\underline{A}$. In both cases a Bayesian estimate based on uniform $P(f)$ and the $d_{m}$ at hand is made of the expected value of $g(\underline{x})=$ $\min _{\bar{x}} f(\underline{x}, \bar{x})$ for each $\underline{x}$. Since we are striving to maximize the worst possible payoff from $f$, the optimal search process selects the move which maximizes this expected value while the worst process selects the move which minimizes this value. More formally, if $E\left(C \mid d_{m}, a, \underline{A}\right)$ differs for the two choices of $\underline{A}$, always being higher for one of them, then $E(C \mid m, a, \underline{A})=\sum_{d_{m}} P\left(d_{m} \mid a\right) E\left(C \mid d_{m}, \underline{A}\right)$ differs for the two $\underline{A}$. In tum, $E(C \mid m, a, \underline{A})=\sum_{f, C}[C \times P(C \mid$ $f, m, a, \underline{A}) \times P(f)] \propto \sum_{f, C}[C \times P(C \mid f, m, a, \underline{A})]$ for the uniform prior $P(f)$. Since this differs for the two $\underline{A}$, so must $\sum_{f} P(C \mid f, m, a, \underline{A})$.

Let $\tilde{g}(\underline{x})$ be a random variable representing the value of $g(\underline{x})$ conditioned on $d_{m}$ and $\underline{x}$, i.e. it equals the worst possible

${ }^{6}$ Recall that $|X|=\sum_{\underline{x}} \bar{l}(\underline{x})$. payoff (to the agent) after the agent makes move $\underline{x}$ and the opponent replies. In the example of section IV we have $\mathbb{E} \tilde{g}(1)=1 / 2$ and $\mathbb{E} \tilde{g}(2)=3 / 4$

To determine the expected value of $\bar{g}(\underline{x})$ we need to know $P\left(\tilde{g}(\underline{x}) \mid \underline{x}, d_{m}\right)=\sum_{f} P\left(\tilde{g}(\underline{x}) \mid \underline{x}, d_{m}, f\right) P(f)$ for uniform $P(f)$. Of the entire population $d_{m}$ only the subset sampled at $\underline{x}$ is relevant. We assume that there are $k\left(\underline{x}, d_{m}\right) \leq m$ such values. ${ }^{7}$ Since we are concerned with the worst possible opponent response let $r\left(\underline{x}, d_{m}\right)$ be the minimal $Y$ value obtained over the $k\left(\underline{x}, d_{m}\right)$ responses to $\underline{x}$, i.e. $r\left(\underline{x}, d_{m}\right)=$ $\min _{\bar{x} \in d_{m}^{\bar{x}}} d_{m}^{y}(\underline{x}, \bar{x})$. Since payoff values are normalized to lie between 0 and $1,0<r\left(\underline{x}, d_{m}\right) \leq 1$. Given $k\left(\underline{x}, d_{m}\right)$ and $r\left(\underline{x}, d_{m}\right), P\left(\tilde{g} \mid \underline{x}, d_{m}\right)$ is independent of $\underline{x}$ and $d_{m}$ and so we indicate the desired probability as $\pi_{k, r}(\tilde{g})$.

In appendix $\mathrm{A}$ we derive the probability $\pi_{k, r}$ in the case where all $Y$ values are distinct (we do so because this results in a particularly simple expression for the expected value of $\tilde{g}$ ) and in the case where $Y$ values are not forced to be distinct. From these densities we the expected value of $\bar{g}(\underline{x})$ can be determined. In the case where $Y$ values are not forced to be distinct there is no closed form for the expectation. However, in the continuum limit where $|Y| \rightarrow \infty$ we fnd (see appendix B)

$$
\mathbb{E}\left(\tilde{g}(\underline{x}) \mid \underline{x}, d_{m}\right)=\frac{1-\left(1-r\left(\underline{x}, d_{m}\right)\right)^{\bar{l}(\underline{x})-k\left(\underline{x}, d_{m}\right)+1}}{\bar{l}(\underline{x})-k\left(\underline{x}, d_{m}\right)+1} .
$$

where we have explicitly noted that both $k$ and $r$ depend both on the move $\underline{x}$ as well as the training population $d_{m}$. As shorthand we defne $C_{m}(\underline{x}) \equiv \mathbb{E}\left(\tilde{g}(\underline{x}) \mid \underline{x}, d_{m}\right)$.

The best move given the training population is the deterministic choice $\underline{A}_{\mathrm{best}}\left(d_{m}\right)=\arg \max _{x} C_{m}(\underline{x})$ and the worst last move is $\underline{A}_{\text {worst }}\left(d_{m}\right)=\arg \min _{x} \bar{C}_{m}(\underline{x})$. In the example of section IV with the population of size $2, \underline{A}_{\text {best }}\left(d_{2}\right)=2$ and $\underline{A}_{\text {worst }}\left(d_{2}\right)=1$.

As long as $C_{m}(\underline{x})$ is not constant (which will usually be the case since the $r$ values will differ) $\left(a, \underline{A}_{\text {best }}\right)$ and $\left(a, \underline{A}_{\text {worst }}\right)$ will differ, and the expected performance of $A_{\text {best }}$ will be superior. This proves that the expected performance over all payoff functions of algorithm $\left(a, \underline{A}_{\text {best }}\right)$ is greater than that of algorithm ( $\left.a, \underline{A}_{\text {worst }}\right)$.

\section{OTHER FREE LUNCHES}

We have shown the existence of free lunches for self-play by constructing a pair of algorithms with the same search rule

${ }^{7}$ Of course, we must also have $k\left(\underline{x}, d_{m}\right) \leq \bar{l}(\underline{x})$ for all populations $d_{m}$. 
$a$ but different move-choosing rules $\underline{A}$ and showing different performance. Unsurprisingly, we can also construct algorithms with different expected performance but having the same move-choosing rule and different search rules. In this section we provide a simple example of such a pair of algorithms. This should help demonstrate that free lunches are a rather common occurrence in self-play settings.

Take the simple case where all $\underline{l}$ agent moves offer the same possible number of opponent responses, say $\vec{l}$. Consider a search rule that explores $m=\underline{l}$ distinct joint samples. Agent moves are labeled by $\underline{x} \in\{1, \cdots \underline{l}\}$ and opponent responses are labeled by $\bar{x} \in\{1, \cdots \bar{l}\}$. For simplicity we assume that $\underline{l}=\bar{l}$.

Search rule $a_{1}$ explores the joint moves $(1,1), \cdots,(1, m)$ while search rule $a_{2}$ explores the joint moves $(1,1), \cdots,(m, 1)$, i.e. $a_{1}$ exhausts opponent responses to $\underline{x}=1$ while $a_{2}$ only samples 1 opponent response to each of it's $m$ possible moves. For the move choosing rule we apply the Bayes optimal iule used earlier: select the move $\underline{x}$ that has the highest expected $g(\underline{x})$ value when averaged over payoff functions consistent with the observed population.

To start we determine the expected performance of an algorithm which does not have the beneft of knowing any opponent responses. In this case we average the performance, $g(\underline{x})$ for any element $\underline{x}$, over all $|Y| \underline{\underline{l}} \underline{l}$ functions. We note that for any given player move $\underline{x}$ the $|Y|^{l \underline{l}}$ possible function values at the joint moves $(\underline{x}, \cdot)$ are replicated $|Y|^{\underline{l} \bar{l}} /|Y|^{\bar{l}}=|Y|^{\bar{l}(\underline{l}-1)}$ times. The number of times that a $g(\underline{x})$ value of $1-i /|Y|$ is attained in the $\mathfrak{f}$ rst $|Y|^{\bar{l}}$ distinct values is $(i+1)^{\bar{l}}-i^{\bar{l}}$. Thus the average $g(\underline{x})$ value, which we denote $\langle g\rangle$, is

$$
\langle g\rangle=\sum_{i=0}^{|Y|-1}\left(1-\frac{i}{|Y|}\right) n_{\bar{l}}(i) .
$$

where $n_{\bar{l}}(i)=[(i+1) /|Y|]^{\bar{l}}-[i /|Y|]^{\bar{l}}$. This average is value is obtained for all moves $\underline{x}$. In the continuum limit where $|Y| \rightarrow \infty$ the expected value of $g$ is simply

$$
\langle g\rangle=1 /(1+\bar{l}) .
$$

This serves as a baseline for comparison; any algorithm which samples some opponent responses has to do better than this.

Next we consider the algorithm $a_{1}$ which exhaustively explores all opponent responses to $\underline{x}=1$. There are $|Y|^{\bar{l}}$ possible populations that this algorithm might see. For each of these populations, $d_{m}$, we need to determine $g(1)$ and the average $g$ values for each of the other moves $\underline{x} \neq 1$. This average is taken over the $|Y|^{\bar{l}(\underline{l}-1)}$ functions consistent with $d_{m}$. Of course we have $g(1)=\min d_{m}^{y}$ and the expected $g(\underline{x})$ value for $\underline{x} \neq 1$ are all equal to $\langle g\rangle$ above. Since the move choosing rule maximizes the expected value of $g$ the expected performance of $a_{1}$ for this population is $\max \left(\min d_{m}^{y},\langle g\rangle\right)$. Averaged over all functions (populations) the expected performance of $a_{1}$ is

$$
\langle g\rangle_{1}=\frac{1}{|Y|^{\bar{l}}} \sum_{d_{m}^{v}} \max \left(\min d_{m}^{y},\langle g\rangle\right)
$$

where the sum is over all $|Y|^{\bar{l}}$ possible populations. Converting the sum over all populations into a sum over the minimum value of the population we fnd

$$
\begin{aligned}
\langle g\rangle_{1} & =\sum_{i=0}^{|Y|-1} \max \left(1-\frac{i}{|Y|},\langle g\rangle\right) n_{\bar{l}}(i) \\
& =\sum_{i=0}^{\lfloor|Y|(1-\langle g\rangle\rangle\rfloor}\left(1-\frac{i}{|Y|}\right) n_{\bar{l}}(i)+\langle g\rangle \sum_{i=\lceil|Y|(1-\langle g\rangle)\rceil}^{|Y|-1} n_{\bar{l}}(i) .
\end{aligned}
$$

If we defne $i_{g} \equiv\lceil|Y|(1-\langle g\rangle)]$ then we obtain

$$
\langle g\rangle_{1}=\sum_{i=0}^{i_{g}-1}\left(1-\frac{i}{|Y|}\right) n_{\bar{l}}(i)+\langle g\rangle\left\{1-\left(\frac{i_{g}}{|Y|}\right)^{\bar{l}}\right\} .
$$

In the continuum limit we have

$$
\begin{aligned}
\langle g\rangle_{1} & =(1-\langle g\rangle)^{\bar{l}} \frac{1+\langle g\rangle \bar{l}}{1+\bar{l}}+\langle g\rangle\left(1-\langle 1-\langle g\rangle)^{\bar{l}}\right) \\
& =\frac{1}{1+\bar{l}}\left(1+\left(\frac{\bar{l}}{1+\bar{l}}\right)^{1+\bar{l}}\right)
\end{aligned}
$$

where we have recalled the expected value $\langle g\rangle=1 /(1+\bar{l})$. We note that as $\bar{l} \rightarrow \infty$ the performance of algorithm $a_{1}$ is $\left(1+e^{-1}\right)$ times that of $\langle g\rangle$.

The analysis of algorithm $a_{2}$ is slightly more complex. In this case each element of the population occurs at a different $\underline{x}$. For any given observed population the optimal move for the player is $\underline{x}^{*}=\arg \max d_{m}^{y \underline{x}}$ (i.e. the move corresponding to the largest payoff observed in the population). With this insight, we observe that when summing over all functions, there are $|Y|^{\bar{l}-1}$ possible completions to $\max d_{m}^{y}$ for the remaining $\bar{l}-1$ unobserved responses to $\underline{x}^{*}$. We must take the minimum over these possible completions to determine the expected value of $g$. Thus the expected payoff for algorithm $a_{2}$ when averaging over all functions (populations) is

$$
\langle g\rangle_{2}=\frac{1}{|Y|^{\bar{l}}} \sum_{d_{m}^{y}} \sum_{i=0}^{|Y|-1} \min \left(\max d_{m}^{y}, 1-\frac{i}{|Y|}\right) n_{\bar{l}-1}(i) .
$$

We proceed in the same fashion as above by defning ${ }^{8} i_{d} \equiv$ $|Y|\left(1-\max d_{m}^{y}\right)$ (which depends on $\left.d_{m}^{y}\right)$ so that

$$
\begin{aligned}
\langle g\rangle_{2} & =\frac{1}{|Y|^{\bar{l}}} \sum_{d_{m}^{y}}\left[\max d_{m}^{y} \sum_{i=0}^{i_{d}-1} n_{\bar{l}-1}(i)+\sum_{i=i_{d}}^{|Y|-1} \frac{|Y|-i}{|Y|} n_{\bar{l}-1}(i)\right] \\
& =\frac{1}{|Y|^{\mid l}} \sum_{d_{m}^{y}}\left[\max d_{m}^{y}\left(\frac{i_{d_{m}^{y}}^{y}}{|Y|}\right)^{\bar{l}-1}+\sum_{i=i_{d_{m}^{y}}^{y}}^{|Y|-1} \frac{|Y|-i}{|Y|} n_{\bar{l}-1}(i)\right]
\end{aligned}
$$

The sum over populations is now tackled by converting it to a sum over the $|Y|$ possible values of $\max d_{m}^{y}$. The number of sequences of length $\bar{l}$ having maximum value $j$ is $j^{\bar{l}}-(j-1)^{\bar{l}}$. Moreover, if $\max d_{m}^{y}=j /|Y|$ then $i_{d}=|Y|-j$ and so

$\langle g\rangle_{2}=\sum_{j=1}^{|Y|}\left[\frac{j}{|Y|}\left\{1-\frac{j}{|Y|}\right\}^{\bar{l}-1}+\sum_{i=|Y|-j}^{|Y|-1} \frac{|Y|-i}{|Y|} n_{\bar{l}-1}(i)\right] n_{\bar{l}}(j-1)$

${ }^{8}$ There is no need to take the ceiling since $i_{d}$ is automatically an integer. 
The continuum limit in this case is found as

$$
\begin{aligned}
\langle g\rangle_{2} & =\bar{l} \int_{0}^{1} d y_{j}\left[y_{j}\left(1-y_{j}\right)^{\bar{l}-1}+(\bar{l}-1) \int_{1-y_{j}}^{1} d y(1-y) y^{\bar{l}-2}\right] y_{j}^{\bar{l}-1} \\
& =\int_{0}^{1} d y_{j} y_{j}^{\bar{l}}\left(1-y_{j}\right)^{\bar{l}-1}+\int_{0}^{1} d y_{j} y_{j}^{\bar{l}-1}-\int_{0}^{1} d y_{j} y_{j}^{\bar{l}-1}\left(1-y_{j}\right. \\
& =B(\bar{l}+1, \bar{l})+1 / \bar{l}-B(\bar{l}, \bar{l})
\end{aligned}
$$

respectively.

In summing the above expressions over $f$ we replace the sum over $f$ with a sum over $j_{1}, j_{2}, k_{1}, k_{2}, l$ and $m$ using the appropriate multiplicities. The resulting sums are then ponverted to integrals in the continuum limit and evaluated by Monte Carlo. Details are presented in Appendix D.

The results are shown in Figure 2 which plots the fraction where $B(x, y)$ is the beta function defned by $B(x, y)=$ $\Gamma(x) \Gamma(y) / \Gamma(x+y)$. For large $\bar{l}$ the Beta functions almost cancel and the expected performance for $a_{2}$ varies as $1 / \bar{l}$ which is only slightly better than the performance of the algorithm which does not have access to any training data.

In Figure 1 we plot the expected performance of $a_{1}$ and $a_{2}$ as a function of $\bar{l}$ (recall that $m=\underline{l}=\bar{l}$ ). Algorithm $a_{1}$ outperforms algorithm $a_{2}$ on average for all values of $\bar{l}$.

Though $a_{1}$ outperforms $a_{2}$ on average, it is interesting to determine the fraction of functions where $a_{1}$ will perform no worse than $a_{2}$. This fraction is given by $|Y|^{-\underline{l} l} \sum_{f} \theta\left(\operatorname{perf}_{1}(f)-\operatorname{perf}_{2}(f)\right)$ where $\operatorname{perf}_{1}(f)$ is the performance of algorithm $a_{1}$ on payoff function $f, \operatorname{perf}_{2}(f)$ is the performance of algorithm $a_{2}$ on the same $f$, and $\theta$ is a step function defned as $\theta(x)=1$ if $x \geq 0$ and $\theta(x)=0$ otherwise. The Bayes optimal payoff for $a_{1}$ for any given payoff function $f$ is $^{9}$

$$
\operatorname{perf}_{1}(f)=\left\{\begin{array}{ll}
\min _{\bar{x}} f(1, \bar{x}) & \text { if } \min _{\bar{x}} f(1, \bar{x})>\langle g\rangle \\
\min _{\bar{x}} f(2, \bar{x}) & \text { otherwise }
\end{array} .\right.
$$

Similarly, the performance of algorithm $a_{2}$ is given by

$$
\operatorname{perf}_{2}(f)=\min _{\bar{x}} f\left(\underline{x}_{2}^{*}, \bar{x}\right) \quad \text { where } \underline{x}_{2}^{*}=\underset{\underline{x}}{\arg \max } d_{m}^{y \mid x} .
$$

To determine the performance of the algorithms for any given $f$ we divide $f$ into its relevant and irrelevant components as follows:

$$
\begin{gathered}
\frac{j_{1}}{|Y|} \equiv f(1,1), \quad \frac{j_{2}}{|Y|} \equiv f(2,1) \\
\frac{k_{1}}{|Y|} \equiv \min _{\bar{x}}\{f(1, \bar{x}) \mid \bar{x} \neq 1\}, \quad \frac{k_{2}}{|Y|} \equiv \min _{\bar{x}}\{f(2, \bar{x}) \mid \bar{x} \neq 1\} \\
\frac{l}{|Y|} \equiv \max _{\underline{x}}\{f(\underline{x}, 1) \mid \underline{x} \neq 1,2\}, \\
\frac{m}{|Y|} \equiv \min _{\bar{x}}\left\{f\left(\underline{x}_{2}^{*}, \bar{x}\right) \mid \underline{x}_{2}^{*} \neq 1,2, \bar{x} \neq 1\right\}
\end{gathered}
$$

In the defnition of $m, \underline{x}_{2}^{*}$ is the move chosen by $a_{2}$ if $a_{2}$ doesn't choose move $\underline{x}_{2}^{*}=1$ or 2 , the specifc value of $\underline{x}_{2}^{*}$ is irrelevant. Given these defnitions, the performance of the two algorithms are

$$
\operatorname{perf}_{1}(f)=\frac{1}{|Y|} \begin{cases}\min \left(j_{1}, k_{1}\right) & \text { if } \min \left(j_{1}, k_{1}\right)>|Y|\langle g\rangle \\ \min \left(j_{2}, k_{2}\right) & \text { otherwise }\end{cases}
$$

and

$$
\operatorname{perf}_{2}(f)=\frac{1}{|Y|} \begin{cases}\min \left(j_{1}, k_{1}\right) & \text { if } \max \left(j_{1}, j_{2}, l\right)=j_{1} \\ \min \left(j_{2}, k_{2}\right) & \text { if } \max \left(j_{1}, j_{2}, l\right)=j_{2} \\ \min (l, m) & \text { otherwise }\end{cases}
$$

${ }^{9}$ We have arbitrarily assumed that $a_{1}$ will select move 2 if it does not select more 1 . This choice has no bearing on the result. of functions for which perf $1 \geq$ perf $_{2}$. This plot was generated using $10^{7}$ Monte Carlo samples per $\bar{l}$ value.

\section{A. Better Training Algorithms}

In the previous sections we constructed Bayes optimal algorithms in limited settings by using specially constructed deterministic rules $a$ and $\underline{A}$. This alone is suffcient to demonstrate the availability of free lunches in self-play contexts. However, we can build on these insights to construct even better (and even worse) algorithms by also determining (at least partially) the Bayes-optimal search rule, $(a, \underline{A})$, which builds out the training set, and selects the champion strategy. That analysis would parallel the approach taken in [MW98] used to study bandit problems. and would further increase the performance gap between the (best, worst) pair of algorithms.

\section{ThE ROLE OF OPPONENT "INTELLIGENCE"}

All results thus far have been driven by measuring performance based on $g(x)=\arg \min _{\bar{x}} f(\underline{x}, \bar{x})$. This is a very pessimistic measure as it assumes that the agent's opponent is omniscient and will make the move most detrimental to the agent. If the opponent is not omniscient and can not determine $\bar{x}^{*}=\arg \min _{\bar{x}} f(\underline{x}, \bar{x})$, how does this affect the availability of free lunches?

Perhaps the simplest way to quantify the intelligence of the opponent is through the fraction, $\alpha$, of payoff values known to the opponent. The opponent will use these known values to estimate its optimal move $\bar{x}^{*}$. We have already examined the $\alpha=1$ limit corresponding to maximal intelligence where the opponent can always determine $\bar{x}^{*}$ and we have free lunches. In the $\alpha=0$ limit the opponent can only make random replies so that the expected performance of the agent will be the average over the opponents possible responses.

One way to approach this problem is to build the opponent's bounded intelligence into the agent's payoff function $g$ and proceed as we did in the omniscient case. If $|X|$ is the number of joint moves, then there are $\left(\begin{array}{c}|X| \\ \alpha|X|\end{array}\right)$ possible subsets of joint moves of size $\alpha|X|{ }^{10}$ We indicate the list of possible subsets as $\mathcal{S}(X, \alpha|X|)$, and a particular subset by $\mathcal{S}_{i} \in \mathcal{S}$. For this particular subset $\bar{x}^{*}$ is estimated by selecting the best response out of the $S_{i}$ payoff values known to the opponent. Of course, it may be the case that there are no samples in $\mathcal{S}_{i}$ having the agent's move $\underline{x}$ and in that case the opponent can only select a random response. In this case the agent will on average obtain the average payoff $\sum_{\bar{x}} f(\underline{x}, \bar{x}) / l(\underline{x})$. If we assume that all subsets of size $\alpha|X|$ are equally likely, then the agent's

\footnotetext{
${ }^{10}$ We assume that $\alpha$ is an integral multiple of $1 /|X|$.
} 


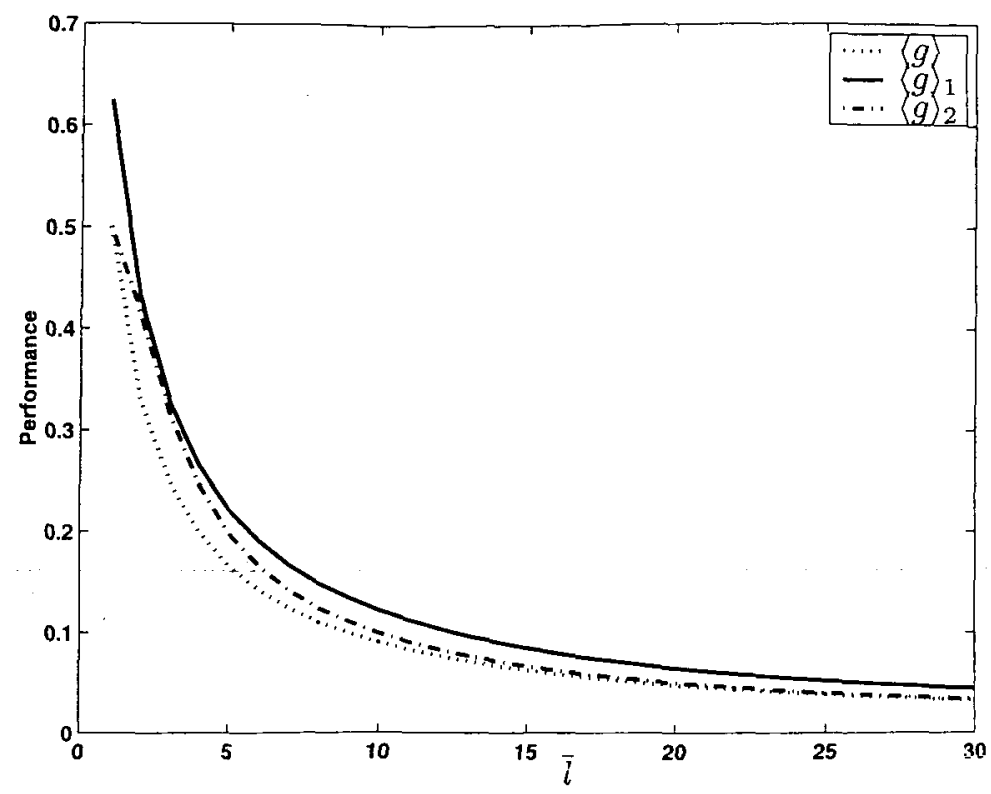

Fig. 1. Expected performance of algorithm $a_{1}$ (indicated as $\left.\langle g\rangle_{1}\right)$ which exhaustively enumerates the opponents response to a particular move, and algorithm $a_{2}$ (indicated as $\langle g\rangle_{2}$ ) which samples only 1 opponent response to each move. For comparison we also plot $\langle g\rangle$ which is the expected performance of an algorithm which does no sampling of opponent responses.

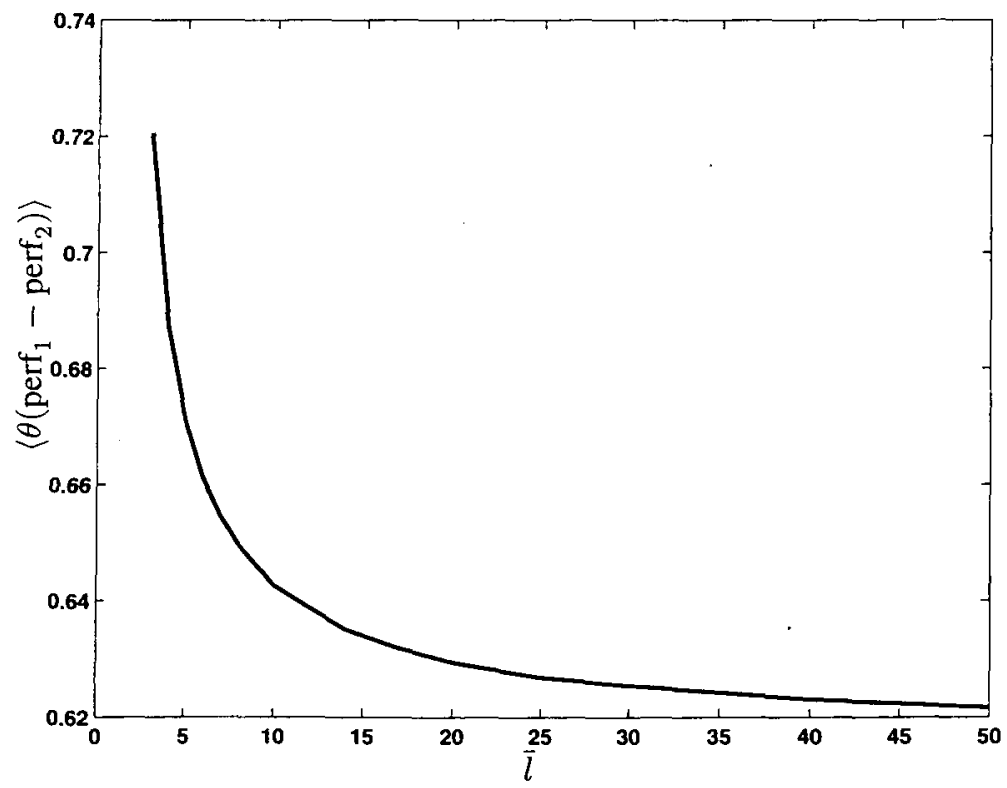

Fig. 2. The fraction of functions in the continuum limit where perf $1 \geq$ perf $f_{2}$ The fgure was generated with $10^{7}$ Monte Carlo samples of the integrand for each value of $\bar{l}$.

payoff function against a boundedly intelligent opponent is given by

$$
g^{\alpha}(\underline{x})=\left(\begin{array}{c}
|X| \\
\alpha|X|
\end{array}\right)^{-1} \sum_{\mathcal{S}_{i} \in \mathcal{S}(X, \alpha|X|)} \underset{(x, \bar{x}) \in \mathcal{S}_{i}}{\arg \min } f(\underline{x}, \bar{x}) .
$$

This generalization reduces to the previously assumed $g$ in the maximally intelligent $\alpha=1$ case. In Table II the functions $g^{1 / 4}, g^{2 / 4}, g^{3 / 4}$, and $g^{4 / 4}$ are listed for the example of section IV. As expected the payoff to the agent increases with decreasing $\alpha$ (a less intelligent opponent). However, we also observe that for the same population $d_{2}$ the average $[g(\underline{x}=1) g(\underline{x}=2)]$ values are $[5 / 8 \quad 7 / 8]$ for $\alpha=1 / 4$, $\left[\begin{array}{ll}29 / 48 & 41 / 48\end{array}\right]^{\top}$ for $\alpha=2 / 4,\left[\begin{array}{ll}9 / 16 & 13 / 16\end{array}\right]^{\top}$ for $\alpha=$ $3 / 4$, and $\left[\begin{array}{ll}1 / 2 & 3 / 4\end{array}\right]^{\top}$ for $\alpha=4 / 4$. For this population, $d_{2}$ ftness function $\left(a, \underline{A}_{\text {best }}\right)$ continues to beat $\left(a, \underline{A}_{\text {worst }}\right)$ and by the same amount independent of $\alpha$.

\section{CONCLUSIONS}

We have introduced a general framework for analyzing NFL issues in a variety of contexts. When applied to selfplay we have proven the existence of pairs of algorithms one of which is superior to another for all possible joint payoff 


\begin{tabular}{|c|c|c|c|c|c|c|c|c|c|c|c|c|c|c|c|c|}
\hline$\alpha$ & $f_{1}$ & $f_{2}$ & $f_{3}$ & $f_{4}$ & $f_{5}$ & $f_{6}$ & $f_{7}$ & $f_{8}$ & $f_{g}$ & $f_{10}$ & $f_{11}$ & $f_{12}$ & $\mathbf{f}_{\mathbf{1 3}}$ & $f_{14}$ & $f_{15}$ & $f_{16}$ \\
\hline $3 / 4$ & $\frac{1}{2}, \frac{1}{2}$ & $\frac{5}{8}, \frac{1}{2}$ & $\frac{5}{8}, \frac{1}{2}$ & $1, \frac{1}{2}$ & $\frac{1}{2}, \frac{5}{8}$ & $\frac{5}{8}, \frac{5}{8}$ & $\frac{5}{8}, \frac{5}{8}$ & $1, \frac{5}{8}$ & $\frac{1}{2}, \frac{5}{8}$ & $\frac{5}{8}, \frac{5}{8}$ & $\frac{5}{8}, \frac{5}{8}$ & $1, \frac{5}{8}$ & $\frac{1}{2}, 1$ & $\frac{5}{8}, 1$ & $\frac{5}{8}, 1$ & 1,1 \\
\hline
\end{tabular}

TABLE II

EXHALSTIVE. ENUMERATION OF ALl 16 POSSIBLE AGENT PAYOFfS, $g^{\alpha}(\underline{x}=1), g^{\alpha}(\underline{x}=2)$, FOR BOUNDEDLY INTELLIGENT OPPONENTS HAVING INTELLIGENCE PARAMETER $\alpha=1 / 4, \alpha=2 / 4, \alpha=3 / 4$, AND $\alpha=4 / 4$. SEE TABLE I FOR THE CORRESPONDING $f$ FUNCTIONS AND FOR THE $\alpha=1 g$ FUNCTION. THE PAYOFF FUNCTIONS LABELED IN BOLD ARE THOSE CONSISTENT WITH THE POPULATION $d_{2}=\{(1,2 ; 1 / 2),(2,2 ; 1)\}$.

functions $f$. This result stands in marked contrast to similar analyses for optimization in non-self-play settings. Basically, the result arises because under a minimax criteria the sum over all payoff functions $f$ is not equivalent to a sum over all functions $\min _{\bar{x}} f(\cdot, \bar{x})$. We have shown that for simple algorithms we can calculate expected performance over all possible payoff functions and in some cases determine the fraction of functions where one algorithm outperforms another. On the other hand, we have also shown that for the more general biological coevolutionary settings, where there is no sense of a "champion" like there is in self-play, NFL still applies

Clearly we have only scratched the surface of an analysis of coevolutionary and self-play optimization. Many of the same questions posed in the traditional optimization setting can be asked in this more general setting. Such endeavors may be particularly rewarding at this time given the current interest in the use of game theory and self-play for multi-agent systems [PW02].

\section{REFERENCES}

[CF99] K. Chellapilla and D. B. Fogel. Evolution, neural networks, games, and intelligence. Proc. IEEE, 87:1471-1498, 1999.

[CK03] D. W. Come and J. D. Knowles. No free lunch and free leftovers theorems for multiobjective optimisation problems. In Second International Conference on Evolutionary Multi-Criterion Optimizarion, pages 327-341. Springer LNCS, 327-341 2003.

[CO01] Steffen Christensen and Franz Oppacher. What can we learn from no free lunch? a frst attempt to characterize the concept of a searchable function. In Lee Spector, Erik D. Goodman, Annie Wu, W.B. Langdon, Hans-Michael Voigt, Mitsuo Gen, Sandip Sen, Marco Dorigo, Shahram Pezeshk, Max H. Garzon, and Edmund Burke, editors, Proceedings of the Genetic and Evolutionary Computation Conference (GECCO-2001), pages 1219-1226, San Francisco, California, USA, 7-11 July 2001. Morgan Kaufmann.

[FHSO1] A. Franz, K. H. Hoffmann, and P. Salamon. Best possible strategy for fnding ground states. Phys. Rev. Lett., 86:5219 - 5222, 2001.

[FPO0] S. G. Ficici and J. B. Pollack. A game-theoretic approach to the simple coevolutionary algorithm. In $\mathrm{M}$. Schoenauer, $\mathrm{K}$. Deb, G. Rudolph, X. Yao, E. Lutton, and H-P.l Schwefel J. J. Merelo, editors, Parallel Problem Solving from Nature VI. Springer Verlag, September 2000.

[Hi192] W. D. Hillis. Coevolving parasites improce simulated evolution as an optimization procedure. In C. G. Langton, C.Taylor, J. D. Farmer, and S. Rasmussen, editors, Artifcial Life II, pages 313322. Addison Wesley, 1992.

[Hol75] J. H. Holland. Adaptation in Natural and Artifcial Systems. MTT Press, Cambridge, MA, 1975.

[IT01] Christian Igel and Marc Toussaint. On classes of functions for which no free lunch results hold. available at http://arXiv. org/abs/physics/0102009, 2001.

[KWM01] M. Koeppen, D.H. Wolpert, and W. G. Macready. Remarks on a recent paper on the 'no free lunch' theorems. IEEE Trans. on Evolutionary Computation, 5(3):295-296, 2001.
[MW96] W. G. Macready and D. H. Wolpert. What makes an optimization problem? Complexity, 5:40-46, 1996.

[MW98] W. G. Macready and D. H. Wolpert. Bandit problems and the exploration/exploitation tradeoff. IEEE Trans. Evol. Comp., 2:222, 1998.

[PW02] S. Parsons and M. Wooldridge. Game theory and decision theory in multi-agent systems. Autonomous Agents and Multi-Agent Systems, 5(3):243-254, Sept 2002.

[SVW01] C. Schumacher, M. D. Vose, and L. D. Whitley. The no free lunch and problem description length. In Lee Spector, Erik D. Goodman, Annie Wu, W. B. Langdon, Hans-Michael Voigt, Mitsuo Gen, Sandip Sen, Marco Dorigo, Shahram Pezeshk, Max H. Gazzon, and Edmund Burke, editors, Proceedings of the Genetic and Evolutionary Computation Conference (GECCO-2001), pages 565-570, San Francisco, California, USA, 7-11 2001. Morgan Kaufmann.

[WM97] D. H. Wolpert and W. G. Macready. No free lunch theorems for optimization. IEEE Trans. Evol. Comp., 1:67-83, 1997.

\section{APPENDIX}

\section{A. Determination of $\pi_{k, r}(\tilde{g}):$ distinct $Y$}

To determine $\pi_{k, r}(\tilde{g})$ we frst consider the case where all $Y$ values are distinct and then consider the possibility of duplicate $Y$ values. Though we only present the non-distinct case in the main text we derive the distinct $Y$ case here because we can obtain a closed form expression for the probability and because it serves as simpler introduction to the case of nondistinct $Y$.

To derive the result we generalize from a concrete example. Consider the case where $|Y|=10, \vec{l}(\underline{x})=5$, and $k=3$. A particular instantiation is presented in Figure 3. In this case $r=4 / 10$ which is not the true minimum for responses to $\underline{x}$. The probability that $r$ is the true minimum is simply $k / \bar{l}(\underline{x})$. If $r$ is not the true minimum then $P\left(\tilde{g} \mid d_{m}\right)$ is found as follows. $P\left(\tilde{g}=1 / 10 \mid d_{m}\right)$ is the fraction of functions containing $Y$ values at $\{1 / 10\} \cup d_{m}^{y \mid x}$. ${ }^{11}$ Since the total number of possibilities consistent with the data is $\left(\begin{array}{l}|Y|-k \\ \bar{l}(\underline{x})-k\end{array}\right)$ this fraction is $\left(\frac{|Y|-k-1}{l(\underline{x})-k-1}\right) /\left(\frac{|Y|-k}{l}(\underline{x})-k\right)=(\bar{l}(\underline{x})-k) /(|Y|-k)$. Similarly, $P\left(\tilde{g}=2 / 10 \mid d_{m}\right)$ is $\left(\frac{|Y|-k-2}{\bar{l}(\underline{x})-k-1}\right) /\left(\begin{array}{l}|Y|-k \\ \bar{l}(\underline{x})-k\end{array}\right)$ because we know that the function can not contain a sample having ftness less than $2 / 10$.

Thus, in the general case, we have

$\pi_{k, r}(\tilde{g})=\left(\begin{array}{l}a \\ b\end{array}\right)^{-1}\left\{\theta(r-\tilde{g})\left(\begin{array}{c}a-|Y| \tilde{g} \\ b-1\end{array}\right)+\delta_{\tilde{g}, r}\left(\begin{array}{c}a-|Y| r+1 \\ b\end{array}\right)\right\}$

${ }^{11}$ By $d_{m}^{y \mid x}$ we mean the set of $Y$ values sampled at $\underline{x}$. 


\begin{tabular}{|c|c|c|c|c|c|c|c|c|c|c|}
\hline$Y$ & $1 / 10$ & $2 / 10$ & $3 / 10$ & $4 / 10$ & $5 / 10$ & $6 / 10$ & $7 / 10$ & $8 / 10$ & $9 / 10$ & $10 / 10$ \\
\hline$f(\underline{x}, \cdot)$ & & & $*$ & $*$ & & $*$ & $*$ & & $*$ & \\
\hline$d_{m}^{y}$ at $\underline{x}$ & & & & $*$ & & $*$ & & & $*$ & \\
\hline$P\left(\tilde{g} \mid d_{m}\right)$ & $6 / 21$ & $5 / 21$ & $4 / 21$ & $6 / 21$ & 0 & 0 & 0 & 0 & 0 & 0 \\
\hline
\end{tabular}

Fig. 3. Row 1 indicates the $Y$ values obtainable on a particular payoff function $f$ for each of the $\bar{l}(\underline{x})=5$ possible opponent responses. Row 2 gives the $Y$ values actually observed during the training period. Row 3 gives the probabilities of $\bar{g}$ assuming a uniform probability density across the $f$ which are consistent with $d_{m}$. The expected value of $P\left(\tilde{g} \mid d_{m}\right)$ is $2.48 / 10$.

where $a=|Y|-k, b=\bar{l}(\underline{x})-k, \theta(x)=1$ iff $x>0$, and $\delta_{\bar{g}, r}=1$ iff $\tilde{g}=r$. Since it is easily veri£ed that

$$
\left(\begin{array}{c}
a-|Y| r+1 \\
b \ldots \ldots \ldots . . .
\end{array}\right)+\sum_{\tilde{g}^{\prime}=1}^{|Y| r-1}\left(\begin{array}{c}
a-\tilde{g}^{\prime} \\
b-1
\end{array}\right)=\left(\begin{array}{l}
a \\
b
\end{array}\right)
$$

this probability is correctly normalized. The expected value of $\tilde{g}$ is therefore

$\mathbb{E}\left(\tilde{g} \mid d_{n}\right)=\left[|Y|\left(\begin{array}{l}a \\ b\end{array}\right)\right]^{-1}\left\{r\left(\begin{array}{c}a-|Y| r+1 \\ b\end{array}\right)+\sum_{\bar{g}^{\prime}=1}^{|Y| r-1} \tilde{g}^{\prime}\left(\begin{array}{c}a-\tilde{g}^{\prime} \\ b-1\end{array}\right)\right\}$

Evaluating this sum we fnd

$$
\begin{aligned}
\mathbb{E}\left(\tilde{g} \mid d_{m}\right) & =\left[|Y|\left(\begin{array}{l}
a \\
b
\end{array}\right)\right]^{-1}\left\{\left(\begin{array}{l}
a+1 \\
b+1
\end{array}\right)-\left(\begin{array}{c}
a+1-|Y| r \\
b+1
\end{array}\right)\right\} \\
& =\frac{|Y|^{-1}}{b+1} \frac{(a+1) \frac{b+1}{b+(a+1-|Y| r)^{\underline{b+1}}}}{a \underline{\underline{b}}}
\end{aligned}
$$

where the falling power, $a^{a}$, is defned by $a^{\underline{b}} \equiv a(a-1)(a-$ 2) $\cdots(a-b+1)$. For the case at hand where $|Y|=10, \bar{l}(\underline{x})=$ 5 , and $k=3$ we have $a=7$ and $b=2$. Since $r=4 / 10$ the expected value is $\mathbb{E}\left(\tilde{g} \mid d_{m}\right)=\frac{1}{10}\left(8^{\underline{3}}-4^{\underline{3}}\right) /\left(3^{3} \cdot 7^{2}\right)=52 / 21 \approx$ $2.48 / 10$.

\section{B. Determination of $\pi_{k, r}(\tilde{g}):$ non-distinct $Y$}

In Figure 4 we present another example where $\bar{l}(\underline{x})=5$, $k=3$, and $r=4 / 10$. In this case however, there are duplicate $Y$ values. The total number of functions consistent with the data is $|Y|^{\bar{l}(\underline{x})-k}=|Y|^{b}$. In this case it is easiest to begin the analysis with the case $\tilde{g}=r$. The number of functions having the minimum of the remaining $b$ points equal to $|Y|$ is 1 . Similarly, the number of functions having a minimum value of $(|Y|-1)$ is $2^{b}-1.2^{b}$ counts the number of functions where the $b$ function values can assume one of $Y$ or $Y-1$. The -1 accounts for the fact that 1 of these functions has a minimum value of $Y$ and not $Y-1$. Generally, the number of functions having a minimum value of $r^{\prime}$ is $\left(|Y|-|Y| r^{\prime}+\right.$ $1)^{b}-\left(|Y|-|Y| r^{\prime}\right)^{b}$. All $r^{\prime} \geq r$ will result in the minimal observed value $r$ so that the total number of functions having an observed minimum of $r$ is

$\sum_{r^{\prime}=r}^{|Y|}\left[\left(|Y|-|Y| r^{\prime}+1\right)^{b}-\left(|Y|-|Y| r^{\prime}\right)^{b}\right]=(|Y|-|Y| r+1)^{b}$.

Thus the probability of $\tilde{g}=r$ is

$$
\pi_{k, r}(\tilde{g}=r)=|Y|^{-b}(|Y|-|Y| r+1)^{b} .
$$

We turn now to determining the probabilities where $\tilde{g}<r$.
Of the $b$ remaining $Y$ values the probability that the minimum is $\tilde{g}$ is

$$
\pi_{k, r}(\tilde{g})=|Y|^{-b}\left\{(|Y|-|Y| \tilde{g}+1)^{b}-(|Y|-|Y| \tilde{g})^{b}\right\} .
$$

Combining these results we obtain the fnal result

$$
\begin{aligned}
\pi_{k, r}(\tilde{g})= & \theta(r-\tilde{g})\left\{\left(1-\tilde{g}+\frac{1}{|Y|}\right)^{b}-(1-\tilde{g})^{b}\right\}+ \\
& \delta_{r, \tilde{g}}\left[1-r+\frac{1}{|Y|}\right]^{b} .
\end{aligned}
$$

Given $\pi_{k, r}(\tilde{g})$ the expectation value of $\tilde{g}$ is found as

$$
\begin{aligned}
\mathbb{E}\left(\tilde{g} \mid d_{m}\right)= & r\left(1-r+\frac{1}{|Y|}\right)^{b}+ \\
& \sum_{\tilde{g}=1 /|Y|}^{r-1 /|Y|} \tilde{g}\left\{\left(1-\tilde{g}+\frac{1}{|Y|}\right)^{b}-(1-\tilde{g})^{b}\right\} \\
= & \sum_{r^{\prime}=1 /|Y|}^{r}\left(1-\left(r^{\prime}-\frac{1}{|Y|}\right)\right)^{b}
\end{aligned}
$$

where we have cancelled appropriate terms in the telescoping sum. If we defne $S_{k}(n) \equiv \sum_{i=1}^{n} i^{k}$ then we can evaluate the last sum to fnd

$$
\mathbb{E}\left(\tilde{g} \mid d_{m}\right)=|Y|^{-b}\left\{S_{b}(|Y|)-S_{b}(|Y|-|Y| r)\right\} .
$$

Though there is no closed form expression for $S_{k}(n)$ a recursive expansion of $S_{k}(n)$ in terms of $S_{j}(n)$ for $j<k$ is

$$
S_{k}(n)=\frac{1}{k+1}\left\{n^{k+1}-\sum_{j=0}^{k-1}(-1)^{k-j}\left(\begin{array}{c}
k+1 \\
j
\end{array}\right) S_{j}(n)\right\} .
$$

The recursion is based upon $S_{0}(n)=n$.

In the concrete case above where $|Y|=10, r=4 / 10$, and $b=2$ the expected value is $\frac{1}{10} 294 / 100=2.94 / 10$.

\section{Continuum Limit}

In the limit where $|Y| \rightarrow \infty$ we can approximate the expectation $E\left(\tilde{g} \mid d_{m}\right)$ given by the sum

$$
E\left(\tilde{g} \mid d_{m}\right)=\sum_{r^{\prime}=1 /|Y|}^{r}\left(1-\left(r^{\prime}-1 /|Y|\right)\right)^{b}=\sum_{r^{\prime}=0}^{r-1 /|Y|}\left(1-r^{\prime}\right)^{b}
$$

by the integral

$$
E\left(\tilde{g} \mid d_{m}\right)=\int_{0}^{r} d r^{\prime}\left(1-r^{\prime}\right)^{b}=\frac{1}{b+1}\left\{1-(1-r)^{b+1}\right\} .
$$

The prediction made by this approximation at $|Y|=10$, $r=4$, and $b=2$ is $2.61 / 10$ as opposed to the correct 


\begin{tabular}{|c|c|c|c|c|c|c|c|c|c|c|}
\hline$Y$ & $1 / 10$ & $2 / 10$ & $3 / 10$ & $4 / 10$ & $5 / 10$ & $6 / 10$ & $7 / 10$ & $8 / 10$ & $9 / 10$ & $10 / 10$ \\
\hline$f(\underline{x}, \cdot)$ & & & $*$ & $*$ & & $* *$ & & & $*$ & \\
\hline$d_{m}^{y}$ at $\underline{x}$ & & & & $*$ & & $* *$ & & & & \\
\hline$P\left(\tilde{g} \mid d_{m}\right)$ & $19 / 100$ & $17 / 100$ & $15 / 100$ & $49 / 100$ & 0 & 0 & 0 & 0 & 0 & 0 \\
\hline
\end{tabular}

Fig. 4. Row 1 indicates the $Y$ values obtainable on a particular payoff function $f$ for each of the $\bar{l}(\underline{x})=5$ possible opponent responses. Row 2 gives the $Y$ values actually observed during the training period. Row 3 gives the probabilities of $\bar{g}$ assuming a uniform probability density across the $f$ which are consistent with $d_{m}$. Note that unlike Fig. 3 there are some duplicate $Y$ values. The expected value of $P\left(\tilde{g} \mid d_{m}\right)$ is $2.94 / 10$.

\begin{tabular}{|c|c|}
\hline$j_{1}$ & 1 \\
$j_{2}$ & 1 \\
$k_{1}$ & $\left(|Y|-k_{1}+1\right)^{\bar{l}-1}-\left(|Y|-k_{1}\right)^{\bar{l}-1}$ \\
$k_{2}$ & $\left(|Y|-k_{2}+1\right)^{\bar{l}-1}-\left(|Y|-k_{2}\right)^{\bar{l}-1}$ \\
$l$ & $l^{\bar{l}-2}-(l-1)^{\bar{l}-2}$ \\
$m$ & $(|Y|-m+1)^{\bar{l}-1}-(|Y|-m)^{\bar{l}-1}$ \\
\hline
\end{tabular}

TABLE III

MULTIPLICITIES OCCURRING WHEN CONVERTING THE SUM OVER $f$ TO A SUM OVER THE ALLOWED VALUES OF $j_{1}, j_{2}, k_{1}, k_{2}, l$, AND $m$.

result of $2.94 / 10$. However, had $|Y|=1000$ and $r=400$ the accurate result is $261.65 / 1000$ while the approximation gives $261.3 / 1000$.

\section{Performance Comparison}

In this appendix we evaluate the fraction of functions for which $a_{1}$ performs better or equal to algorithm $a_{2}$ where $a_{1}$ and $a_{2}$ are defned as in Section VI.

The function $\theta\left(\operatorname{perf}_{1}(f)-\operatorname{perf}_{2}(f)\right)$ is equal to 1 if

$$
c d_{1}+e_{1} c d_{2}+e_{2} c \bar{d}_{1} \bar{d}_{2}+\bar{e}_{1} \bar{c} d_{1}+\bar{c} d_{2}+e_{3} \bar{c}_{1} \bar{d}_{1}
$$

where $c=\left(\min \left(j_{1}, k_{1}\right)>|Y|\langle g\rangle\right), d_{1}=\left(\max \left(j_{1}, j_{2}, l\right)=\right.$ $\left.j_{1}\right), d_{2}=\left(\max \left(j_{1}, j_{2}, l\right)=j_{2}\right), e_{1}=\left(\min \left(j_{1}, k_{1}\right) \geq\right.$ $\left.\min \left(j_{2}, k_{2}\right)\right), e_{2}=\left(\min \left(j_{1}, k_{1}\right) \geq \min (l, m)\right), e_{3}=$ $\left(\min \left(j_{2}, k_{2}\right) \geq \min (l, m)\right)$. In the above Boolean expression we have used the condensed notation $a b \equiv a \wedge b, a+b \equiv a \vee b$, and $\bar{a}=\neg a$. It is convenient to factor the Boolean expression as

$$
c\left(d_{1}+e_{1} d_{2}+e_{2} \bar{d}_{1} \bar{d}_{2}\right)+\bar{c}\left(\bar{e}_{1} d_{1}+d_{2}+e_{3} \bar{d}_{1} \bar{d}_{2}\right) .
$$

To give the fraction of functions where $a_{1}$ performs better than $a_{2}$ this expression is to be summed over $j_{1}, j_{2}, k_{1}, k_{2}$, $l$, and $m$ with appropriate multiplicities. The multiplicities are given in Table $\mathrm{III}$.

In the continuum limit this sum becomes the integral

$$
\begin{aligned}
& \int_{0}^{1} d j_{1} \int_{0}^{1} d j_{2} \int_{0}^{1} d k_{1} P\left(k_{1}\right) \int_{0}^{1} d k_{2} P\left(k_{2}\right) \int_{0}^{1} d l P(l) \times \\
& \int_{0}^{1} d m P(m)\left\{c\left(d_{1}+e_{1} d_{2}+e_{2} \bar{d}_{1} \bar{d}_{2}\right)+\bar{c}\left(\bar{e}_{1} d_{1}+d_{2}+e_{3} \bar{d}_{1} \bar{d}_{2}\right)\right\}
\end{aligned}
$$

where $P\left(k_{1}\right)=(\bar{l}-1)\left(1-k_{1}\right)^{\bar{l}-2}, P\left(k_{2}\right)=(\bar{l}-1)\left(1-k_{2}\right)^{\bar{l}-2}$, $P(l)=(\bar{l}-2) l^{\bar{l}-3}, P(m)=(\bar{l}-1)(1-m)^{\bar{l}-2}$, and condition $c$ is modifed to $\min \left(j_{1}, k_{1}\right)>\langle g\rangle$. Though this integral is diffcult to evaluate analytically, it is straightforward to evaluate by Monte Carlo importance sampling of $\left(j_{1}, j_{2}, k_{1}, k_{2}, l, m\right)$ using the respective probability distributions. Samples from $P(u)=m(1-u)^{m-1}$ are obtained by sampling values $v$ from $U(0,1)$ and transforming so that $u=1-v^{1 / m}$; samples from $P(w)=m w^{m-1}$ are obtained via $w=v^{1 / m}$. 